\title{
Les angles morts des travaux sur l'inclusion scolaire à la lumière de la théorie de la justice sociale de Nancy Fraser
}

\author{
Marjorie Vidal, Université du Québec à Montréal \\ Marilyne Boisvert, Université du Québec à Trois-Rivières \\ Flavio Murahara, Université de McGill \\ Corina Borri-Anadon, Université du Québec à Trois-Rivières \\ France Beauregard, Université de Sherbrooke
}

DOI : 10.51186/journals/ed.2021.11-1.e398

\section{Résumé}

Cet article a pour objectif d'éclairer les angles morts des travaux sur l'inclusion scolaire à la lumière de la théorie de la justice sociale de Nancy Fraser. Sur la base des paradigmes de redistribution, de reconnaissance et de représentation, il met en lumière plusieurs angles morts, tant sur le plan politique que paradigmatique. Si l'on n'en tient pas compte, ces angles morts peuvent entrainer une forme normative et prescriptive de l'inclusion à travers la simplification de la complexité du réel, et la mise en place de remèdes correctifs. II convient donc d'adopter une approche critique de l'inclusion, qui peut être facilitée par l'utilisation de certains concepts comme le climat scolaire, la participation sociale et l'advocacy.

Mots-clés : approche critique, inclusion scolaire, justice sociale, redistribution, représentation

\begin{abstract}
This article aims to examine the blind spots of the literature on inclusive education in the light of Nancy Fraser's social justice theory. Based on the paradigms of redistribution, recognition and representation, it identifies several blind spots, located at both the political and paradigmatic levels. If ignored, they can lead to a normative and prescriptive road to inclusion by simplifying the complexity of reality and providing corrective remedies. A critical approach to the concept of inclusion should therefore be adopted, which can be facilitated by the use of concepts such as school climate, social participation and advocacy.
\end{abstract}

Keywords: critical approach, redistribution, representation, school inclusion, social justice 


\section{INTRODUCTION}

Selon le Rapport mondial de suivi sur l'éducation «Inclusion et éducation : tous, sans exception " (Organisation des Nations Unies pour l'éducation, la science et la culture [UNESCO], 2020), l'inclusion scolaire est définie comme un processus qui implique des changements systémiques pour supprimer les obstacles à l'équité et à la participation de tous/tes les élèves, deux finalités interdépendantes (Borri-Anadon, et al., 2015). Le rapport situe ses réflexions dans l'objectif plus large de l'Agenda 2030, soutenu par une volonté de justice sociale. Malgré cette volonté explicite, la théorie de la justice sociale demeure peu mobilisée dans les travaux sur l'inclusion scolaire. De plus, certain-es pratiques et processus éducatifs, dont le repérage des besoins des élèves (Noël, 2019) et les modèles d'organisation des services (réponse à l'intervention ou RAl, et services multipaliers, par exemple) peuvent s'éloigner des visées de justice sociale (Noël, 2019 ; Trépanier, 2019a ; 2019b). C'est en ce sens que certaines pratiques à visées inclusives peuvent être traversées par des processus d'exclusion (Borri-Anadon, et al., 2019).

La prémisse sur laquelle repose cette contribution est qu'il existe un écart entre les postulats du paradigme inclusif et ses applications pratiques, qui pourrait être en partie expliqué par des angles morts de certains travaux sur l'inclusion scolaire. En ce sens, les angles morts recouvrent des éléments théoriques et empiriques souvent invisibilisés, et qui contribuent à rendre excluantes des pratiques pensées comme étant inclusives. À l'heure où plusieurs pays s'inspirent des recommandations internationales de l'UNESCO pour la mise en place de leurs politiques éducatives, il nous semble judicieux de mieux cerner ces angles morts, c'est-à-dire de les mettre en lumière dans une perspective de transformation sociale. L'idée derrière cet exercice est d'éviter certaines "dérives » de l'inclusion scolaire qui peuvent nuire à sa visée de transformation sociale (Bauer \& Borri-Anadon, 2021). L'exercice est d'autant plus important qu'un paradigme inclusif qui ne soutient pas « l'évolution des services aux élèves vers plus de justice sociale et d'équité, pourrait au contraire amener à maintenir le statu quo sous la nouvelle étiquette de l'inclusion " (Prud'homme \& Ramel, 2016, p. 16). La question de la justice sociale permet d'orienter et de penser les politiques et pratiques éducatives inclusives au regard de la visée d'une société plus juste (Clark, 2006). Le paradigme de justice sociale de Nancy Fraser offre à cet effet un éclairage pertinent par le prisme des enjeux de redistribution, de reconnaissance et de représentation.

Selon cette perspective, le présent article propose une réflexion critique exploratoire des travaux scientifiques sur l'inclusion scolaire. À partir d'un regard critique centré sur une démarche de déconstruction/reconstruction (Borri-Anadon, 2014), il poursuit deux objectifs complémentaires : 1) dégager les angles morts des travaux sur l'inclusion scolaire à la lumière des politiques de redistribution, de reconnaissance et de représentation de la théorie de la justice sociale, en reprenant le cadre proposé par Nancy Fraser ; 2) discuter de certains concepts qui pourraient atténuer ces angles morts. Pour atteindre ces deux objectifs, nous 
mettons tout d'abord de l'avant certaines limites des travaux sur l'inclusion scolaire, pour ensuite présenter la théorie de la justice sociale de Nancy Fraser. L'éclairage des dimensions analytiques de cette théorie nous permet de dégager deux types d'angles morts : les angles morts d'ordre politique et d'ordre paradigmatique. Enfin, nous présentons certains concepts qui offrent des pistes de réflexion pour penser l'inclusion de manière critique.

\section{PROBLÉMATIQUE : LES LIMITES DES TRAVAUX SCIENTIFIQUES SUR L'INCLUSION SCOLAIRE}

L'inclusion est un concept populaire que l'on retrouve aussi bien dans les médias, dans les politiques publiques, dans le milieu associatif que dans plusieurs domaines professionnels (Bouquet, 2015). En éducation, l'inclusion est devenue un concept incontournable, largement mobilisé dans la littérature scientifique, dans l'action publique et dans le langage professionnel (Barrère \& Mairesse, 2016; Beauregard \& Trépanier, 2010 ; Bouquet, 2015), « comme un aspect du langage politiquement correct » (Bonvin, et al., 2013, p. 128). Tant et si bien que certain-es parlent d'injonction, de dogme (Bonvin, et al., 2013 ; Pawloff, 2020), voire de l'idéologie de l'inclusion (Bouquet, 2015). C'est pourquoi plusieurs en appellent à la prudence vis-à-vis d'un concept valise, à la rhétorique parfois illusoire (Kauffman \& Hallahan, 1995). Elles et ils recommandent d'adopter une perspective critique, pour mesurer la réelle portée de l'inclusion, dont les principes peuvent être édulcorés ou récupérés politiquement (Goyer \& Borri-Anadon, 2019 ; Larochelle-Audet, 2018).

Parallèlement, plusieurs auteurs/trices soulignent l'inconsistance conceptuelle et les nombreuses imprécisions entourant la définition même de l'inclusion. Elles et ils pointent ainsi une notion « floue et aux significations incertaines » et qui est « [d]'abord pensée par défaut » (Barrère \& Mairesse, 2016, pp. 46-47), c'est-à-dire par référence à son corollaire l'exclusion (Evans \& Lunt, 2002) alors même que le concept d'exclusion fait lui-même l'objet de plusieurs critiques pour son caractère élastique, multidimensionnel et polysémique (Billette, 2012 ; Billette, et al., 2012). II est vrai que le concept d'inclusion a subi de nombreuses mutations au cours des années. Et les termes pour le qualifier se sont multipliés. Dans leur étude sociohistorique, Beauregard et Trépanier (2010) relèvent ainsi les multiples appellations de l'inclusion au cours des années : « intégration, mainstreaming, environnement le moins restrictif (least restrictive environment) (LRE), initiative de l'insertion en enseignement ordinaire (regular education initiative) (REI), inclusion ou inclusion partielle (inclusion, partial inclusion), inclusion totale (full inclusion), pédagogie inclusive, inclusive school, etc. » (p. 33). Cette multiplication des termes entraîne une certaine complexité à appréhender avec acuité le concept d'inclusion sur le plan sémantique.

Ce flou sémantique peut donner lieu à de nombreux problèmes de compréhension qui concourent à creuser un écart entre l'inclusion comme principe idéologique et comme orientation pragmatique (Bauer \& Borri-Anadon, 2021; Beauregard \& Trépanier, 2010 ; Evans 
\& Lunt, 2002 ; Prud'homme, et al., 2016). Plusieurs auteurs/trices déplorent par ailleurs de multiples malentendus, dont le manque de cohérence ainsi qu'une certaine confusion autour de l'inclusion qui peut être à la fois pensée comme un moyen, comme un mouvement ou bien comme une fin en soi, une destination (Barrère \& Mairesse, 2016 ; Beauregard \& Trépanier, 2010 ; Booth, et al., 2003). Si ces malentendus contribuant au flou sémantique ne sont pas toujours visibles d'entrée de jeu, la théorie de la justice sociale de Nancy Fraser permet justement de les mettre en lumière. Ce cadrage analytique est d'autant plus pertinent que très peu d'articles mobilisent cette théorie en éducation (Vincent, 2019).

\section{LA THÉORIE DE LA JUSTICE SOCIALE DE NANCY FRASER}

L'éducation est au cœur des questions de justice sociale, en ce sens qu'elle peut être à la fois une cause et une solution aux inégalités (Power, 2012), dans un contexte où les luttes pour la reconnaissance de groupes subordonnés occupent largement l'espace public. Si la théorie critique de Nancy Fraser (2011) ne se situe pas au centre des questionnements en éducation, elle présente toutefois un intérêt pour analyser les injustices sociales qui s'y déroulent (Vincent, 2019). II s'agit d'une théorie qui dévoile les mécanismes de production des injustices socioéconomiques, des injustices culturelles ou symboliques et des injustices politiques. Pour Nancy Fraser (2009; 2011), le fait d'atteindre la justice sociale est un processus s'attachant à vaincre ces types d'injustice, par l'entremise de politiques de redistribution, de reconnaissance et de représentation. Son paradigme se décline ainsi en trois dimensions interreliées.

D'un côté, le paradigme de la redistribution a trait aux luttes qui concernent les injustices socioéconomiques, telles que la marginalisation, l'exploitation et le dénuement (Fraser, 2011). Ces injustices appellent à deux types de "remèdes ", soit des remèdes correctifs et des remèdes transformateurs. Les remèdes correctifs constituent avant tout des interventions qui ne s'attaquent pas aux causes profondes des injustices. Remettant peu en question l'ordre social, ils cherchent surtout à atténuer les symptômes de ces injustices, par le biais d'une justice redistributive (programme d'assurance-emploi, aide sociale, allocations).

Les remèdes transformateurs, quant à eux, visent le changement et la déconstruction, plus précisément une réorganisation de la structure socioéconomique et, ultimement, une élimination du statut de subordination des groupes ou des classes. Par exemple, dans un cadre scolaire, ce paradigme permet d'appréhender les inégalités entre élèves en termes de différences entre classes sociales : les inégalités de réussite reposeraient ainsi sur une mauvaise répartition des ressources en éducation selon l'appartenance sociale des élèves (Power, 2012). Dans cette perspective, l'inclusion peut être appréhendée à travers la réallocation des ressources selon une perspective compensatoire, voire corrective ou réformiste, mais aussi et surtout comme un outil de remise en question des structures scolaires visant à transformer les inégalités scolaires et sociales. 
D'un autre côté, le paradigme de la reconnaissance se rattache aux revendications qui naissent d'injustices culturelles ou symboliques, telles que la domination culturelle, l'invisibilisation ou le mépris des identités et des productions culturelles des groupes sociaux subordonnés. II peut exiger le recours à des remèdes correctifs, comme des politiques d'affirmative action, qui, par leur fonction performative, (re)créent les groupes. Mais ce paradigme se rattache également à des remèdes transformateurs qui visent la modification des modèles sociaux d'interprétation, de représentations et de communication des identités de tout un chacun, ce qui mène plutôt à la déconstruction de ces groupes (Fraser, 2011).

Appliqué dans un cadre scolaire, ce paradigme amène à s'intéresser aux mécanismes de désignation de certains groupes d'élèves, comme les élèves issu-es de l'immigration, les élèves que l'on nomme "en situation de handicap " ou d'autres que l'on étiquette "à risque ", ainsi qu'aux mécanismes de catégorisation leur attribuant des "difficultés » ou des " besoins » particuliers. Selon cette perspective, les inégalités de réussite dont sont victimes ces groupes sont liées à des impositions culturelles, comme la nature ethnocentrée du curriculum. Et l'inclusion pourrait prendre la forme d'une déconstruction des catégories, à travers la reconnaissance et la valorisation des identités multiples et fluides, selon une perspective de transformation sociale.

Pour complexifier son modèle qui se veut initialement dualiste (reconnaissance/redistribution), Nancy Fraser (2009) préconise également le recours au concept de représentation politique, qui illustre la manière dont les injustices politiques peuvent compromettre la parité participative dans les arènes sociales et politiques où les discours se créent, circulent, se reconstruisent. Pour le dire autrement, les injustices politiques sont celles qui marginalisent ; elles empêchent certains groupes d'avoir une voix dans différentes arènes dont celles où sont prises les décisions quant aux politiques de reconnaissance et de redistribution.

Pour remédier à ces injustices, Nancy Fraser (2011) suggère d'opérer une transformation institutionnelle ou structurelle, du local, en passant par le national, jusqu'au transnational afin que les institutions soient érigées en véritables instances démocratiques. On pourrait donc dire que, dans ce cadre, les inégalités scolaires touchent ce qui empêche des élèves (voire leurs parents) de participer à la vie éducative sur le plan politique. Afin de supprimer ces inégalités, une perspective inclusive exige alors d'adopter une approche ascendante (bottom-up) et de repenser certains dispositifs mis en place dans le dessein de favoriser ou maintenir la représentation et la réelle participation de certains groupes d'élèves (et parents) dans les arènes de décisions éducatives (conseil scolaire, implication au conseil d'établissement, etc.).

À la lumière de la théorie de la justice sociale de Nancy Fraser, la mise en place de l'inclusion nécessite à la fois redistribution, reconnaissance et représentation : elle implique d'assurer un accès équitable aux ressources, mais aussi de limiter le pouvoir des structures scolaires qui maintiennent la méritocratie, d'accorder une place aux groupes subordonnés, mais aussi de questionner leur essentialisation au sein de l'espace scolaire d'œuvrer pour que l'école soit le 
reflet de la diversité de la société dont elle est issue, mais aussi d'être à l'écoute des contrediscours. Toutefois, l'opérationnalisation de ces exigences est marquée par une grande complexité, compte tenu des multiples tensions qui traversent les travaux sur l'inclusion scolaire. La théorie de la justice sociale de Nancy Fraser peut justement servir à analyser certains angles morts des travaux sur l'inclusion scolaire.

\section{LES ANGLES MORTS DES TRAVAUX SUR L'INCLUSION SCOLAIRE}

Les paradigmes de redistribution, de reconnaissance et de représentation de la théorie de la justice sociale (Fraser, 2011) peuvent être mobilisés comme des perspectives analytiques, permettant d'étudier en profondeur des situations sociales susceptibles de créer des injustices socioéconomiques, culturelles et symboliques dans les travaux sur l'inclusion scolaire. Cet éclairage analytique permet de dégager deux catégories interdépendantes : les angles morts d'ordre politique et les angles morts d'ordre paradigmatique.

\subsection{Les angles morts d'ordre politique : l'invisibilisation des singularités}

Plusieurs des angles morts des travaux sur l'inclusion scolaire se situent au niveau politique. Alors que l'inclusion est à l'agenda de nombreux systèmes éducatifs dans le monde, une grande majorité des travaux qui y réfèrent reprennent la définition de l'UNESCO, sur les bases de la déclaration de Salamanque (1994). Certes, il importe de circonscrire l'inclusion et d'en définir les paramètres généraux, non seulement dans une optique d'opérationnalisation, mais également de cohérence avec la pédagogie scolaire déployée (Prud'homme, et al., 2011). Toutefois, une définition unique et transmise «par le haut » (top-down) peut poser plusieurs problèmes qui sont révélés par l'éclairage des paradigmes de redistribution, de reconnaissance et de représentation de la théorie critique de Nancy Fraser.

Une des dérives à cet égard pourrait être de vouloir offrir à tout prix l'école inclusive à tous/ tes les élèves. En effet, l'éclairage du paradigme de redistribution révèle que l'inclusion, telle qu'elle s'opérationnalise dans cet exemple, n'est pas toujours la réponse adéquate. Les représentations de l'inclusion dépendent entre autres des difficultés attribuées aux élèves, de leur vécu, de leur progression dans le cursus scolaire (Evans \& Lunt, 2002 ; Goransson, 2009 ; Mesibov \& Shea, 1996), mais également des pratiques enseignantes (Beauregard, 2015 ; Cook, 2004; Kabano, 2000). À cela s'ajoute l'idée qu'une définition unique de l'inclusion induit qu'il y ait certaines pratiques qui soient acceptables (et qui conduiraient à une inclusion « réussie ") et d'autres qui ne le sont pas.

Cette perspective prescriptive, qui fait écho aux principes d'une forme scolaire normative, empêche parallèlement l'émergence d'autres modèles ou pratiques plus marginales qui viseraient également l'inclusion. Cette définition pourrait ainsi contribuer à exclure les personnes qui ne s'intègrent pas selon les normes prescrites d'inclusion ou qui s'intègrent 
différemment ${ }^{1}$ (pour toutes sortes de raisons), reproduisant ainsi une forme d'exclusion de certains groupes. Toute définition de l'inclusion est sujette à interprétation. Le fait de ne pas tenir compte des singularités propres aux milieux, des systèmes éducatifs en place, etc., ne permet pas de considérer que les dispositifs d'inclusion peuvent être très variables d'un endroit à l'autre. Dès lors, on peut s'interroger quant à savoir si une définition unique de l'inclusion peut recouvrir la diversité des situations (Nguyen et al., 2015 ; Symeonidou, 2017 ; Zuany, 2018).

La question est d'autant plus prégnante puisque toute politique s'inscrit dans un espace et une temporalité spécifiques, et fait l'objet de multiples interprétations qui sont susceptibles d'en transformer l'énoncé formel initial en resignifiant les finalités et les moyens dans les pratiques (Ball, 1997 ; Maroy, et al, 2013). C'est ainsi que, par exemple, les politiques d'adaptation scolaire ont beaucoup évolué au fil du temps et sont appliquées de façon très différente entre les pays, les organisations scolaires, voire entre les écoles (Carlson Berg, 2011). Au Québec, certains milieux favorisent les approches mainstreaming ou d'inclusion partielle, alors que d'autres privilégient l'approche d'inclusion totale (Beauregard \& Trépanier, 2010). Comme autre exemple québécois, on peut penser à la Politique d'intégration scolaire et d'éducation interculturelle (Ministère de l'Éducation du Québec [MEQ], 1998), dont les diverses modalités de mise en œuvre ont subi diverses transformations depuis son adoption (Ministère de l'Éducation, du Loisir et du Sport, 2014 ; Ministère de l'Éducation et de l'Enseignement Supérieur, 2020).

Par ailleurs, le fait que l'inclusion repose sur une transmission « par le haut » (top-down), c'est-à-dire qu'elle se transmet par le prisme des politiques éducatives, entraîne une relégation des principales/aux concerné-es. Les élèves (et les parents, mais plus généralement tous-tes les acteurs/trices de la communauté éducative) qui sont visé-es par l'inclusion se voient alors confisquer leur statut de membre de la société, comme sur la base de modèles normatifs auxquels elles et ils n'ont pas ou peu participé (Fraser, 2004) et dont elles et ils n'en comprennent souvent ni les règles, ni le sens (Vidal, 2019). À l'inverse, une approche qui se dit inclusive gagnerait à être initiée "par le bas » (bottom-up), afin de favoriser la participation de tous/tes les acteurs/trices de la communauté éducative pour que les élèves et leurs parents puissent se subjectiver sur le plan individuel et collectif.

\subsection{Les angles morts d'ordre paradigmatique : l'invisibilisation des marqueurs}

D'autres angles morts des travaux sur l'inclusion scolaire se situent au niveau paradigmatique : ils révèlent la tension entre l'invisibilisation et l'amplification des marqueurs de la diversité, comme le genre, l'orientation sexuelle, la religion, etc. Cette tension est perceptible à travers la façon dont ces marqueurs sont appréhendés dans les travaux sur l'inclusion scolaire.

\footnotetext{
1 On peut penser notamment au développement d'écoles par et pour certains groupes sociaux ou encore à des choix de scolarisation parallèle.
} 
Certains travaux s'intéressent aux pratiques ou aux modèles d'organisation des services inclusifs sans égard aux différents marqueurs de la diversité ou sans considération particulière aux injustices que les élèves sont susceptibles de vivre en raison de ces marqueurs ${ }^{2}$. Se voulant fédérateurs, ces travaux sont ceux qui portent sur tous/tes les élèves. Leurs retombées consistent à diluer les frontières intergroupes, et ainsi à éliminer toute étiquette ou tout stigmate. D'un autre côté, d'autres travaux s'attachent plutôt à rendre visibles ces marqueurs. Leurs apports résident dans l'idée de donner une voix à des individus marginalisé-es ou subordonné-es ; ils concourent à rendre compte des réalités complexes de divers groupes sociaux et à mettre en lumière les failles des pratiques ou des modèles d'organisation des services qui peuvent exclure certains groupes en particulier. Même si les chercheurs/ses derrières ces deux «types » de travaux se réclament du paradigme inclusif, des dérives relatives à leur posture sont possibles.

En ce qui concerne le premier groupe de travaux (invisibilisation des marqueurs), le fait d'occulter les différents marqueurs de la diversité nuit à la portée de l'inclusion. Cette approche implique que la diversité n'est pas l'apanage de groupes subordonnés ou marginalisés, mais désigne presque toute différence entre les individus (Sanders \& BelghitiMahut, 2011). Ces différences individuelles peuvent être perçues comme des traits spécifiques des élèves, traits qui en font leur unicité, alors que leurs différences gagneraient à être valorisées (Prud'homme, et al., 2011). Dans cette perspective, les différences sont considérées comme "égales » et la tendance est à mettre l'accent sur celles-ci plutôt que sur les inégalités.

Le Prévost (2011) estime qu'un tel usage de la diversité est à craindre puisqu'il tend à gommer « le caractère asymétrique et antagonique des rapports sociaux à l'origine de ces discriminations pour n'en faire que des relations, toutes au même niveau, et qu'il resterait à pacifier et à harmoniser » (p. 62). En faisant fi du fait que certaines « différences » sont plus stigmatisantes que d'autres, les voix des populations désavantagées sont susceptibles de se noyer dans celles de populations privilégiées. Bien que l'on cherche, à travers cette perspective, à mieux comprendre comment mettre en œuvre des pratiques qui déconstruisent les catégories socioscolaires, le risque demeure d'occulter les multiples rapports de force qui se jouent en société. Par ricochet, on peut oublier que les injustices socioéconomiques et culturelles ou symboliques sont construites au travers de rapports sociaux, qu'elles coexistent et se renforcent dialectiquement, comme nous le rappelle Nancy Fraser (2011).

Le deuxième groupe de travaux sur l'inclusion (reconnaissance des marqueurs de la diversité) prend le contrepied en s'attardant spécifiquement à certains marqueurs de la diversité. II s'agit par exemple de travaux qui mobilisent des concepts liés aux « besoins particuliers » et

\footnotetext{
2 Dans la majorité des écrits sur l'inclusion scolaire, le public cible est les élèves en adaptation scolaire (les élèves dits handicapés ou en difficulté d'adaptation ou d'apprentissage - HDAA) (Prud'homme, et al., 2016), alors que l'inclusion devrait porter sur l'ensemble des élèves et pas seulement les élèves HDAA.
} 
qui permettent de faire entendre des contre-discours, et donc de valoriser cette diversité. Pourtant, ces travaux perpétuent des catégorisations socioscolaires en étiquetant certains élèves. En favorisant la diffusion de stéréotypes ou d'images simplifiées et homogènes de certains groupes, ils peuvent verser dans une perspective psychomédicale ou déficitariste. Pour le dire autrement, se centrer sur des groupes spécifiques peut contribuer à réifier les groupes subordonnés ou les essentialiser, exacerber les frontières intergroupes et ainsi « geler les antagonismes qu'elle[s] cherche[nt] à vaincre » (Fraser, 2011, p. 73).

\subsection{L'inclusion : vers une nouvelle forme de normalisation?}

Les angles morts révélés dans cette partie font écho à un questionnement de Nancy Fraser (2011), qui se demande comment arriver à surmonter les contradictions relatives à la reconnaissance sans tomber dans des dérives autoritaires et normatives. Si la solution ne réside pas uniquement dans les remèdes correctifs, mais dans la transformation des rapports de reconnaissance entre les groupes, on est en droit de se demander comment déconstruire les groupes sans d'abord s'y intéresser pour mieux comprendre ce qui crée leur exclusion. Que ce soit au niveau politique ou paradigmatique, l'éclairage apporté par la théorie de la justice sociale de Nancy Fraser révèle que si l'on ne porte pas attention aux angles morts, une approche inclusive peut reproduire une certaine forme scolaire normative et prescriptive qui va à l'encontre de ses visées initiales.

Parallèlement, l'étude de ces différents angles morts ouvre la réflexion sur la difficulté à concilier une approche qui tend à dénormaliser l'inclusion scolaire tout en permettant une reconnaissance de la diversité des représentations des élèves et de leurs familles sur les processus éducatifs. Afin de se situer dans une perspective de justice sociale, l'inclusion ne peut donc faire l'économie de la complexité du réel et doit tenir compte de la participation des différent-es acteurs/trices qu'elle vise. Certains concepts, lorsqu'ils sont employés dans une perspective de justice sociale, peuvent favoriser ces deux éléments. Ils sont développés dans la section suivante.

\section{DES CONCEPTS QUI OFFRENT QUELQUES PISTES DE RÉFLEXION SUR L'INCLUSION SCOLAIRE}

Plutôt que de nous arrêter à la critique des travaux sur l'inclusion scolaire, dans le cadre de cet article, nous avons pris le parti de privilégier une avenue plus constructive et de susciter une réflexion sur des pistes prometteuses. Certains concepts afférents de l'inclusion sont en effet porteurs des enjeux de justice sociale soulevés dans cet article et permettent de penser l'inclusion de manière critique, selon la perspective transmise par la théorie de Nancy Fraser. Ils mettent ainsi l'accent sur la diversité des situations et sur la participation des acteurs/trices scolaires. II s'agit des concepts de climat scolaire, de participation sociale et d'advocacy. Mais ces concepts ne doivent pas être épargnés de leur propre examen. En effet, une compréhension partielle de ce qu'ils sous-tendent pourrait générer des paradoxes ou être à 
l'origine d'une mise en œuvre superficielle, ce qui les inscrirait alors en porte-à-faux de l'approche de justice sociale.

\subsection{Le climat scolaire}

Selon une perspective sociale, pour qu'il y ait inclusion, il faut que le réel soit pris en compte dans toute sa complexité. Le climat scolaire est un concept qui offre justement une meilleure appréhension du contexte. II est défini comme la résultante d'interactions entre les individus et entre les individus et leur environnement (Janosz, et al., 1998). II recouvre plusieurs dimensions, dont les attitudes, les pratiques et les politiques, sur lesquelles reposent les interactions présentes dans un établissement scolaire (Archambault, et al., 2018). Le climat scolaire cherche notamment à éclairer l'arrimage entre instruction et socialisation au sein de l'expérience scolaire.

Pour parvenir à cet arrimage, les chercheurs/ses qui le mobilisent peuvent avoir recours à des concepts connexes, comme ceux de communauté et de réussite éducatives. Ce faisant, elles et ils sont appelé-es à rendre visible la manière dont la mobilisation de la première (communauté) amène à définir la seconde (réussite), selon les enjeux propres au contexte à l'étude. Le climat scolaire se définit de l'intérieur : les expériences des individus contribuent au climat, et inversement. Dans ce sens, il permet d'identifier celles et ceux dont l'expérience est traversée par des injustices culturelles ou symboliques, et ainsi mobiliser les catégories du social plus à même de les révéler. II contribue ainsi à identifier quels groupes sont surinvisibilisés, et ainsi susciter des réflexions permettant de tendre vers la déconstruction de ces catégories.

Toutefois, si le climat scolaire permet de reconnaître des groupes et de s'interroger sur les rapports entre ceux-ci, il n'a pas toujours le potentiel transformateur nécessaire dans une perspective de justice sociale. Afin de prendre acte de cette limite, il importe de comprendre l'expérience scolaire comme le résultat de dynamiques systémiques, institutionnelles et interindividuelles. Dans cette perspective, le climat scolaire dépasse la seule perspective individuelle comme facteur explicatif du social et interpelle l'école en tant que communauté d'acteurs/trices. II peut donc être mobilisé pour appréhender la manière dont les injustices se déploient dans l'établissement scolaire et étudier les remèdes mis en œuvre pour les combattre. Par exemple, par le biais des concepts de zones de vulnérabilité et de pratiques d'équité, Archambault, et al. (2019) ont mis en lumière des processus et pratiques à effet d'exclusion, mais également la nature corrective des remèdes développés. Somme toute, si le concept de climat scolaire n'est pas transformateur en lui-même, il permet de jeter les bases d'une réflexion collective de l'ensemble de la communauté éducative à cet égard. 


\subsection{La participation sociale et l'advocacy}

Il existe de nombreuses définitions de la participation sociale dans la littérature scientifique. Par-delà leurs divergences, les chercheurs/ses semblent s'accorder sur trois points qui touchent à la fois le volet individuel et sociétal de la participation sociale : 1) la personne effectue une activité ; 2) cette activité lui permet d'interagir avec les autres ; et 3) cette activité lui permet de contribuer à la société (Larrivière, 2008 ; Levasseur, et al., 2010). Dans un cadre scolaire, il s'agit par exemple d'un-e élève qui participe à des travaux d'équipe. Elle ou il effectue une activité qui lui permet d'interagir avec d'autres personnes et de contribuer à l'équipe. La participation sociale émane de la personne qui demande une reconnaissance et une prise en compte de son potentiel de la part des différents-es acteurs/trices. Elle veut s'accomplir, pas seulement agir, à travers les activités qu'elle effectue et que cette participation soit reconnue comme un apport précieux par la société. Cette personne considère qu'elle est la mieux placée pour se représenter. Elle connait ses forces, ses besoins, et son potentiel, que la société est à même d'entendre.

Pourtant, ces définitions réfèrent peu à la valorisation du rôle de la personne dans cette activité. C'est en ce sens que la question de l'engagement est centrale dans l'appréhension de la participation sociale, ancrée dans une perspective de justice sociale. II est donc essentiel de réfléchir à l'engagement des personnes pour s'éloigner d'une approche prescriptive et normative de l'inclusion qui renforcerait les angles morts mentionnés dans cet article. L'approche de la justice sociale selon Nancy Fraser implique que la participation sociale dépasse la simple action - interaction - contribution. Elle valorise le rôle de la personne dans cette activité : celle-ci voit son potentiel reconnu par la société tout en ayant conscience de ce qu'elle peut lui apporter (Beauregard, 2015 ; Beauregard, et al., 2019; Nye-Lengerman \& Hewitt, 2019).

À l'instar du concept de participation parentale, l'advocacy réfère à l'expression du point de vue et l'implication des personnes. Toutefois, l'advocacy se situe à un niveau plus global que la participation sociale et implique d'emblée un changement systémique en faisant référence au soutien public pour un changement de situation ou de politique. l'advocacy ou selfadvocacy (l'auto-représentation) (Wehmeyer \& Shogren, 2017) est en lien avec le développement de compétences telles que le leadership, la communication, la connaissance de soi et la connaissance de ses droits (Test, et al., 2005). Ce concept est d'autant plus pertinent lorsqu'il concerne des groupes marginalisés et touche à leurs possibilités de s'exprimer (Mitchell, 2006).

Dans un cadre scolaire, ce concept fait référence à l'expression du point de vue des élèves ou des membres de la communauté (parents et personnel) sur les possibles améliorations dans une école. En ce sens, l'advocacy appréhende les revendications des parents ou des élèves comme des voix réduites au silence (silenced), voire dénaturées (misrepresented) par 
l'organisation scolaire, surtout en ce qui concerne les prises de décisions, qu'elles soient organisationnelles ou curriculaires. Les établissements d'enseignement, selon cette perspective, deviennent des lieux fondamentaux pour lutter contre les injustices et les barrières qui empêchent la participation paritaire (Keddie, 2012). Toutefois, les pratiques d'advocacy ne garantissent pas nécessairement la promotion de la justice sociale (Keddie, 2012). C'est ainsi qu'un intérêt de groupe particulier peut prévaloir sur d'autres groupes marginalisés, comme lors des réunions scolaires, où les voix des adultes ne reflètent pas toujours les voix des élèves (Black, 2011). Dans cette optique, Nancy Fraser (2009) offre une lentille critique pour tenir compte de ces inégalités, en s'intéressant à la représentation des points de vue et de la complexité de la parité participative.

\section{CONCLUSION}

Dans le cadre de cet article, nous avons étudié certains angles morts du concept d'inclusion à la lumière de la théorie de la justice sociale de Nancy Fraser, soit à travers les paradigmes de redistribution, de reconnaissance et de représentation. L'exercice nous a permis de révéler une possible dérive de certains travaux scientifiques sur l'inclusion scolaire. Celle-ci s'incarne dans une forme normative et prescriptive de l'inclusion : dans la simplification de la complexité du réel (situations, représentations, participation, marqueurs, etc.) et dans la mise en place de remèdes correctifs qui occultent les rapports de pouvoir sous-jacents. Cette dérive s'actualise aussi bien sur le plan politique que sur le plan paradigmatique. Notre réflexion nous a ensuite porté-es à considérer certains concepts, tels que le climat scolaire, la participation sociale et l'advocacy, comme des leviers pour une remise en question de la façon dont l'inclusion est étudiée, définie et mobilisée dans la recherche. Ces concepts permettent de traquer l'injustice sociale en ce sens qu'ils suscitent une réflexion sur la prise en compte des singularités des situations et de la participation des acteurs/trices visé-es par l'inclusion. Toutefois, il importe d'adopter la même démarche critique vis-à-vis de ces concepts que celle proposée pour l'inclusion, afin de ne pas accentuer les angles morts révélés dans le cadre de cet exercice.

Cette prise de conscience nous amène à penser que l'adoption d'une approche critique de l'inclusion est nécessaire afin de ne verser ni dans un dogmatisme aveugle, ni dans son rejet total qui serait tout aussi irrationnel. En filigrane, cela soulève la nécessaire analyse du rapport à la norme des chercheurs/ses, qui font face aux limites de leurs propres interprétations. Elles et ils gagneraient à s'interroger sur leurs postures, leurs concepts, voire leurs propres définitions de l'inclusion à l'aune de la théorie de la justice sociale de Nancy Fraser. Et pourraient ainsi se poser certaines questions en amont de leurs recherches : Comment définir et circonscrire la notion d'inclusion dans les recherches ? Comment identifier les conditions de l'inclusion ? Qu'est-ce qui ne fait pas partie de l'inclusion ? Comment considérer les diverses expériences des acteurs/trices de l'inclusion (parents et élèves) ? Quelle place accorder à leurs représentations de l'inclusion ? Incidemment, cela impliquerait 
d'étudier les nombreux liens qui se tissent entre chercheurs/ses et acteurs/trices de l'inclusion scolaire, de comprendre comment elles/ils s'influencent mutuellement, par le prisme de la justice sociale. Car c'est au prix de critiques et autocritiques que des principes comme l'inclusion peuvent survivre sans devenir des dogmes ou des évidences.

\section{RÉFÉRENCES}

Archambault, I. Audet, G., Borri-Anadon, C., Hirsch, S., Mc Andrew, M., \& Tardif-Grenier, K. (2019). L'impact du climat interculturel des établissements sur la réussite éducative des élèves issus de l'immigration. Montréal, QC : Fonds de recherche du Québec - Société et culture (FRQSC). https://frq.gouv.qc.ca/app/uploads/2021/04/ isabellearchambault_rapport_prs_2016-2017_reussite-immigrants.pdf

Archambault, I., Mc Andrew, M., Audet, G., Borri-Anadon, C., Hirsch, S., Amiraux, V. \& TardifGrenier, K. (2018). Vers une conception théorique multidimensionnelle du climat scolaire interculturel. Alterstice, 8(2), 119-135.

Ball, S. J. (1997). Policy sociology and critical social research: a personal review of recent education policy and policy research. British educational research journal, 23(3), 257-274.

Barrère, A., \& Mairesse, F. (2015). L'inclusion sociale. Les enjeux de la culture et de l'éducation. Paris : L'Harmattan.

Bauer, S., \& Borri-Anadon, C. (2021). De la reconnaissance à l'invisibilisation : une modélisation des enjeux conceptuels de la diversité en éducation inclusive. Alterstice, 10 (2), 45-56. https://www.journal.psy.ulaval.ca/ojs/index.php/ARIRI/article/view/ Bauer_Alterstice10\%282\%29.

Beauregard, F. (2015). Pratiques d'enseignement auprès des élèves dysphasiques dans le contexte d'intégration scolaire au Québec. In J.-C. Kalubi, \& L. Gremion (Eds), Intégration scolaire et nouveaux défis pour la formation des enseignants (pp. 81-102). Montréal, QC : Édition Les Nouvelles.

Beauregard, F., Chrysagis, M., Charbonneau, J. \& Couture, M. (2019, August 6). Views of Adults with ASD of social participation. International Association for the Scientific Studies of Intellectual and Developmental Disabilities. Glascow, Écosse.

Beauregard, F., \& Trépanier, N. (2010). Le concept d'intégration scolaire... mais où donc se situe l'inclusion? In N. Trépanier, \& M. Paré (Eds.), Des modèles de service pour favoriser l'intégration scolaire (pp. 31-56). Québec : Presses de l'Université du Québec.

Billette, V. (2012). D'une société exclusive à une société inclusive et plurielle : Perspectives de solidarités en gérontologie sociale. In J.-P. Lavoie, \& N. Guberman (Eds), Les cahiers du CREGÉS.

http://www.creges.ca/wp-content/uploads/2018/10/2012_3_Cahier-du-CREGES_FR.pdf

Billette, V., Lavoie, J.-P., Séguin, A.-M., \& Van Pevenage, I. (2012). Réflexions sur l'exclusion et l'inclusion sociale en lien avec le vieillissement. L'importance des enjeux de reconnaissance et de redistribution. Frontières, 25(1), 10-30. 
Black, R. (2011). Student participation and disadvantage: limitations in policy and practice. Journal of Youth Studies, 14(4), 463-474.

\section{https://doi.org/10.1080/13676261.2010.533756}

Bonvin, P., Ramel, S., Curchod-Ruedi, D., Albanese, O., \& Doudin, A. (2013). Inclusion scolaire : de l'injonction sociopolitique à la mise en œuvre de pratiques pédagogiques efficaces. Alter, 7(2), 127-34.

Booth, T., Nes, K., \& Strømstad, M. (2003). Developing inclusive teacher education. London: Routledge Falmer.

Borri-Anadon, C. (2014). Pratiques évaluatives des orthophonistes à l'égard des élèves issus de minorités culturelles : une recherche interprétative-critique. Thèse de doctorat inédite, Faculté des sciences de l'éducation, Université du Québec à Montréal. https:// archipel.uqam.ca/6351/1/D2628.pdf

Borri-Anadon, C., Bergeron, G, Point, M., \& Letscher, S. (2019). Éditorial : Comprendre l'exclusion pour mieux parvenir à l'inclusion? / Understanding exclusion to better work towards inclusion? McGill Journal of Education / Revue des sciences de l'éducation de McGill, 54(2), 186-193.

Bouquet, B. (2015). L'inclusion : approche socio-sémantique. Vie sociale, 11(3), 15-25.

Carlson Berg, L. (2011). Un regard critique sur les initiatives d'éducation inclusive des élèves immigrants en milieu scolaire fransaskois. Francophonies d'Amérique, (32), 65-86.

Clark, J. A. (2006). Social justice, Education and Schooling: Some Philosophical Issues, British Journal of Educational Studies, 54(3). Social Justice and Education: New and Continuing Themes, 272-287.

Cook, B. (2004). Inclusive teachers' attitudes toward their students with disabilities: A replication and extension. The Elementary School Journal, 104(4), 307-320.

Evans, J., \& Lunt, I. (2002). Inclusive education: are there limits? European Journal of Special Needs Education, 17(1), 1-14.

Fougeyrollas, P., Cloutier, R., Bergeron, H., Côté, J., Côté, M., \& St-Michel, G. (1996). Révision de la proposition québécoise de classification : Processus de production du handicap. Québec : Comité québécois sur la Classification internationale des déficiences, incapacités et handicaps.

Fraser, N. (2009). Scales of Justice. Reimagining political space in a globalizing world. New York, NY: Columbia University Press.

Fraser, N. (2004). Justice sociale, redistribution et reconnaissance. Revue du MAUSS, 1(1), 152-164.

Fraser, N. (2011). Qu'est-ce que la justice sociale ? Reconnaissance et redistribution. Paris : Éditions La découverte.

Göransson, K. (2009). L'inclusion : différentes perspectives différentes significations. La nouvelle revue de l'adaptation et de la scolarisation, 2(2), 11-16.

Goyer, R., \& Borri-Anadon, C. (2019). Le paradigme inclusif à travers le prisme des rapports sociaux inégalitaires. McGill Journal of Education / Revue des sciences de l'éducation de McGill, 54(2), 194-205. 
Janosz, M., Georges, P., \& Parent, S. (1998). L'environnement éducatif à l'école secondaire : un modèle théorique pour guider l'évaluation du milieu. Revue Canadienne de PsychoÉducation, 27(2), 285-306.

Kabano, J. (2000). Les représentations sociales d'enseignantes et d'enseignants des classes ordinaires du primaire à propos des élèves handicapés. Montréal, QC : Université du Québec à Rimouski/Université du Québec à Montréal, Rimouski.

Kauffman, J. M., \& Hallahan, D. P. (1995). The illusion of full inclusion. Austin, TX: Pro-Ed.

Keddie, A. (2012). Schooling and social justice through the lenses of Nancy Fraser. Critical Studies in Education, 53(3), 263-279.

Larivière, N. (2008). Analyse du concept de la participation sociale : définitions, cas d'illustration, dimensions de l'activité et indicateurs. Revue Canadienne d'Ergothérapie, 75(2), 114-127.

Larochelle-Audet, J. (2018). Conceptions de la diversité ethnoculturelle dans la formation en enseignement au Québec : un exercice de mise en visibilité des rapports sociaux de race. Éducation et francophonie, 46(2), 73-91.

Le Prévost, M. (2010). Hétérogénéité, diversité, différences : vers quelle égalité des élèves ? Nouvelle revue de psychosociologie, (1), 55-66.

Levasseur, M., Richard, L., Gauvin, L., \& Raymond, E. (2010). Inventory and Analysis of Definitions of Social Participation Found in the Aging Literature: Proposed Taxonomy of Social Activities. Social Science \& Medicine, 71, 2141-2149.

Maroy, C., Mathou, C., Vaillancourt, S., \& Voisin, A. (2013). 'La Gestion axée sur les résultats' au Québec : récits d'action publique, intérêts des acteurs et médiations institutionnelles dans la fabrication 19 d'une politique éducative. Montréal, QC : Chaire de recherche du Canada en politiques éducatives, Université de Montréal. http://crcpe.umontreal.ca/ publications/documents/RapportNew-AGE_Axe1_FINAL.pdf

Mesibov, G. B., \& Shea, V. (1996). Full inclusion and students with autism. Journal of Autism and Developmental Disorders, 26(3), 337-346.

Ministère de l'Éducation et de l'Enseignement supérieur du Québec (MEES) (2020). Soutien au milieu scolaire 2020-2021. Intégration et réussite des élèves issus de l'immigration et éducation interculturelle. Éducation préscolaire, enseignement primaire et enseignement secondaire. http://www.education.gouv.qc.ca/fileadmin/site_web/documents/education/ diversite/Guide-soutien-milieu-scolaire_2020-2021.pdf

Ministère de l'Éducation, du Loisir et du Sport (MELS). (2014). Rapport d'évaluation. Politique d'intégration scolaire et d'éducation interculturelle. Québec, QC : Gouvernement du Québec.

Ministère de l'Éducation du Québec (MEQ). (1998). Politique d'intégration scolaire et d'éducation interculturelle. Québec, QC : Gouvernement du Québec.

Mitchell, D. (2006). Exploring experiences of advocacy by people with learning disabilities: testimonies of resistance. London: Jessica Kingsley.

Nguyen, X. T. (2015). The Journey to Inclusion. Rotterdam: Sense Publishers. 
Noël, I. (2019). Signaler un élève pour répondre à ses besoins particuliers ou soulager le système ? Revue des sciences de l'éducation de McGill, 54(2), 226-244.

Nye-Lengerman, K. M., \& Hewitt, A. S. (2019). Community living and participation: A comprehensive framework. In A. S. Hewitt' \& K. M. Nye-Lengerman (Eds.), Community living and participation for people with intellectual and developmental disabilities (pp.1-25). Silver Spring: American Association on Intellectual and Developmental Disabilities.

Organisation des Nations Unies pour l'éducation, la science et la culture (UNESCO). (2020). Rapport mondial sur l'éducation 2020. Inclusion et éducation : tous sans exception. https://ccic-unesco.org/unesco-rapport-mondial-sur-leducation-gem-2020-inclusion-eteducation-tous-sans-exception/

Pawloff, S. (2020). Ce que l'inclusion ne dit pas de l'inclusion: Remarques d'anthropologie psychanalytique sur la condition postmoderne des politiques d'inclusion. Empan, 1(1), 21-27.

Power, S. (2012). Redistribution, reconnaissance et représentation : parcours de la lutte contre l'injustice sociale et des changements de politique éducative. Éducation et sociétés, 28, 27-44.

Prud'homme, L., \& Ramel, S. (2016). Introduction. In L. Prud'homme, H. Duchesne, P. Bonvin, \& R. Vienneau (Eds.). L'inclusion scolaire: ses fondements, ses acteurs et ses pratiques (pp.15-17). Louvain : De Boeck.

Prud'homme, L., Vienneau, R., Ramel, S., \& Rousseau, N. (2011). La légitimité de la diversité en éducation : réflexion sur l'inclusion. Éducation et francophonie, 39(2), 6-22.

Sanders, H., \& Belghiti-Mahut, S. (2011). La diversité dans I'Hexagone : les usages français d'une notion américaine. Humanisme et entreprise, 305, 21-36.

Symeonidou S. (2017). Initial teacher education for inclusion: a review of the literature. Disability \& Society, 32(3), 401-422.

Test, D. W., Fowler, C. H., Brewer, D. M., \& Wood, W. M. (2005). A content and methodological review of self-advocacy intervention studies - results of a content and methodological review of the literature of 25 self-advocacy intervention studies are discussed. Exceptional Children: Journal of the International Council for Exceptional Children, 72(1), 101-125.

Trépanier, N. (2019a). La réponse à l'intervention et l'offre de services multipaliers pour soutenir l'inclusion scolaire : un paradoxe à visée d'exclusion des élèves en situation de handicap. Revue des sciences de l'éducation de McGill, 54(2), 284-304.

Trépanier, N. (2019b). Des modèles de service d'orthopédagogie. Montréal : Éditions JFD.

Vidal, M. (2019). De la fabrication de l'échec scolaire en milieu défavorisé et multiethnique.

Revue des sciences de l'éducation de McGill, 54(2), 206-225.

Vincent, C. (2019). Nancy Fraser, Social Justice and Education. London: Routledge.

Wehmeyer, M., \& Shogren, K. (2017). Problem solving and self-advocacy. In Shogren, K. A., Wehmeyer, M. L., \& Singh, N. N. (Eds). Handbook of positive psychology in intellectual and developmental disabilities: translating research into practice (pp. 217 -231). New York, NY: Springer. 
Zuany, R.G.M. (2018). Inclusión como política educativa: hacia un sistema educativo único en um México cultural y lingüísticamente diverso. Sinética, 50, 1-16. 